\title{
Comparative evaluation and economic potential of ecorational versus chemical insecticides for crucifer flea beetle (Coleoptera: Chrysomelidae) management in canola
}

\section{Authors: Frank B. Antwi, D. L. Olson, and J. J. Knodel}

This is a pre-copyedited, author-produced PDF of an article accepted for publication in Economic Entom following peer review. The version of record *see citation below, is available online at: https://doi.org/10_1093/jee/100.3710.

Antwi, Frank B., D. L. Olson and J. J. Knodel. 2007. Comparative evaluation and economic potential of ecorational versus chemical insecticides for crucifer flea beetle (Coleoptera: Chrysomelidae) management in canola. Journal of Economic Entomology 100(3): 710-716. doi: 10.1093/jee/100.3.710 


\title{
Comparative Evaluation and Economic Potential of Ecorational Versus Chemical Insecticides for Crucifer Flea Beetle (Coleoptera: Chrysomelidae) Management in Canola
}

\author{
F. B. ANTWI ${ }^{1}$ D. L. OLSON ${ }^{2}$ AND J. J. KNODEL ${ }^{3}$
}

\begin{abstract}
From 2001 to 2004, field studies were conducted to evaluate the effect of the ecorational insecticides SpinTor (spinosad), BotaniGard (Beauveria bassiana), Neemix (azadirachtin), and Surround (kaolin) against crucifer flea beetle on canola, Brassica napus L., at the cotyledon stage. The ecorational treatments were compared with a standard foliar chemical insecticide, Capture (bifenthrin), and the chemical seed treatment insecticide Helix XTra (thiamethoxam). This study indicated that flea beetle injury was lower for Helix XTra, Capture, and the ecorational insecticide SpinTor. SpinTor was less effective when flea beetle populations were relatively high (200-300 per trap-week). Yields for chemical insecticide treatments were always greater than SpinTor, with differences being the smallest $(68-374 \mathrm{~kg} / \mathrm{ha})$ at low levels of flea beetle feeding injury. Differences were greatest when canola seedling injury was high $(775-1,364 \mathrm{~kg} / \mathrm{ha})$. Yield differences between the conventional insecticides and BotaniGard, Neemix, and Surround were 119-439 and 61-2,248 kg/ha at low and high flea beetle feeding injury, respectively. Although yield differences between SpinTor and chemical insecticides were relatively small at lower levels of flea beetle injury, net losses ranged from $\$ 47$ to $\$ 151 /$ ha when SpinTor was used as an alternative to a standard chemical seed treatment, Helix XTra. This suggests that SpinTor would not be a viable alternative to the chemical insecticide. Net losses ranged from $\$ 30$ to $\$ 266 /$ ha when BotaniGard, Neemix, and Surround were used as alternatives to the seed treatment.
\end{abstract}

KEY WORDS canola, flea beetle, insecticides, injury, yield

Crucifer flea beetle, Phyllotreta cruciferae (Goeze) (Coleoptera: Chrysomelidae), is a key economic pest of canola, Brassica napus L., in the Northern Great Plains of the United States and Canada (Brown 1967, Burgess 1977, Lamb 1984). Adult flea beetles emerge from overwintering sites early in the spring as air temperatures warm to $14-20^{\circ} \mathrm{C}$ (Lamb 1983). Beetles move into canola fields and immediately begin feeding on young cotyledons and leaves, which results in reduced seedling plant stands within a few days (Westdal and Romanow 1972, Wylie 1979, Lamb 1983). Adults feeding at the cotyledon stage of the crop accounts for the greatest crop loss to this pest (Putnam 1977, Lamb and Turnock 1982, Lamb 1984, Weiss et al. 1991).

Management of crucifer flea beetle is targeted at adults in early spring when the canola crop is in its seedling stage, the most vulnerable stage to flea beetle injury (Thomas 2003). Chemical insecticides, either as

${ }^{1}$ Department of Land Resources and Environmental Sciences, Montana State, University, Bozeman, MT 59717.

${ }^{2}$ Corresponding author: North Dakota Department of Agriculture and Department of Entomology, North Dakota State University, Fargo, ND 58105 (e-mail: deniseolson@nd.gov).

${ }^{3}$ Department of Entomology, North Dakota State University, Fargo, ND 58105. seed treatments or foliar sprays, are the first line of defense against $P$. cruciferae, (Lamb and Turnock 1982). Foliar chemical insecticides are effective against crucifer flea beetle, but only within a narrow window of opportunity; therefore, the majority of canola acreages in the Northern Great Plains are planted to insecticide-treated seed (Turnock and Turnbull 1994, Glogoza et al. 2002). The decision to plant insecticide-treated seed is made before knowledge of a potential flea beetle population and before economic feeding injury occurs. This prophylactic reliance on chemical insecticide-based pest management increases the risk for crucifer flea beetle populations to develop resistance to these materials. The use of ecorational-based insecticides applied in alternative treatment regimes or in combination with reduced rates of conventional insecticides may prevent or delay insecticide resistant development in crucifer flea beetle in canola. The potential for insects to develop resistance to chemical insecticides, coupled with a focus in agriculture for environmentally friendly pest management, drives the need to evaluate alternative insecticides that could be included in pest management programs for crucifer flea beetle.

Ecorational insecticides are products that are ecologically rational, with no or minimal effects on non- 
target organisms or the environment (Ware 1989). Ecorational insecticides have been effective against numerous insect pests (Hajek et al. 1987, Miranpuri et al. 1992, Miranpuri and Khachatourians 1995, Sparks et al. 1999, Thompson et al. 1999, Gaugler 2004), and they may have potential in crucifer flea beetle management. Ecorational insecticides have unique modes of action compared with chemical insecticides (Sparks et al. 2001, Thompson et al. 2000), and they could play an important role in insecticide resistance management and in environmental conservation (Liu and Stansly 1995, Copping and Menn 2000). Ecorational insecticides have not been tested in the field for crucifer flea beetle control in canola. The objectives of this research were to 1) evaluate ecorational versus chemical insecticide effects on crucifer flea beetle feeding injury to seedling canola and resulting yields; and 2) determine the economic potential of substituting ecorational materials for chemical insecticides to control crucifer flea beetle in canola.

\section{Materials and Methods}

In field studies, entomopathogens, a plant-derived defense chemical, and an antifeedant were compared with a standard foliar chemical and a seed treatment for efficacy to control P. cruciferae. Treatments were arranged in a randomized complete block design with four replications. Research plots, previously seeded to wheat, were established from 2001 through 2004 at North Dakota Agricultural Experiment Station-Research Extension Centers (NDAES-REC) located in Minot (north central), Langdon (northeastern) and Carrington (central) North Dakota, all of which are canola production areas.

Brassica napus Hybrid Roundup Ready (357 RR) seed was planted in early to mid-May. Experimental plots were 1 by $6 \mathrm{~m}$, and they were seeded at $\approx 178$ pure live seeds per $\mathrm{m}^{2}(3.63 \mathrm{~kg} / 0.4 \mathrm{ha})$ with seven rows per plot and seeding depth of $2.5 \mathrm{~cm}$. A single 1-m-wide (single planter pass) buffer plot was included between each treatment plot. This minimized cross-contamination of the spray applications and edge effects of flea beetle invasions. We acknowledge, however, that small plot effects could influence our results that can vary from commercial fields. The foliar chemical insecticide Capture 2 EC (bifenthrin, FMC Corp., Princeton, NJ) was applied at $0.51 \mathrm{ml} /$ liter. The chemical seed treatment Helix XTra (thiamethoxam, Syngenta Crop Protection Inc., Greensboro, NC) was added to the experiment in 2002 and applied at 15.03 $\mathrm{ml} / \mathrm{kg}$ seed. Helix XTra-treated seed was seeded at the same time as the foliar treatments. Seed for the foliar treatments and the control were treated with the fungicide contained in the Helix XTra seed treatment. Ecorational insecticides SpinTor 2 SC (spinosad, Dow Agrosciences, Indianapolis, IN), BotaniGard ES (Beaweria bassiana, Emerald BioAgriculture Corp., Lansing, MI), Surround WP (kaolin, a clay, Engelhard Corp., Iselin, NJ) and Neemix 4.5 EC (azadirachtin, Certis USA, L.L.C., Columbia, MD) were applied as foliar applications at low, medium, and high label rates to individual treatment plots. The rates used were SpinTor: low rate, $1.56 \mathrm{ml} /$ liter; medium rate, 2.34 $\mathrm{ml} /$ liter; and high rate $3.13 \mathrm{ml} /$ liter; BotaniGard: low rate, $6.25 \mathrm{ml} /$ liter; medium rate, $9.37 \mathrm{ml} /$ liter; and high rate, $12.5 \mathrm{ml} /$ liter; Neemix: low rate, $1.56 \mathrm{ml} /$ liter; medium rate, $3.13 \mathrm{ml} /$ liter; and high rate, $6.25 \mathrm{ml} /$ liter; and Surround: low rate, $37.45 \mathrm{~g} /$ liter; medium rate, $93.46 \mathrm{~g} /$ liter; and high rate, $149.8 \mathrm{~g}$ / liter. Spray volume was 189 liters/ha. All foliar applications were applied using a $\mathrm{CO}_{2}$ backpack sprayer after arrival of flea beetles and when canola was in the cotyledon to one-leaf stage. Untreated plots served as the control. Before foliar applications, each plot was rated for flea beetle feeding injury. Along a 4.6-m section of row, plants nearest to $0.3-\mathrm{m}$ intervals were selected for a total of 10 plants. These plants were rated using an injury rating scheme of $1,0-3$ pits per seedling; $2,4-9$ pits; $3,10-15$ pits; $4,16-25$ pits; $5>25$ pits; and 6 , dead seedling (Nowatzki and Weiss 1997).

Residual activity of treatment applications was determined by postapplication ratings for flea beetle injury at 7 and $14 \mathrm{~d}$ after the application date for foliar insecticides. The canola crop was swathed from each whole plot at $30 \%$ seed moisture, and seed yield (kilograms per hectare) was collected from each experimental unit at $8-10 \%$ seed moisture in mid-August. Percentage of oil of yield was determined for harvested seed at 8 to $10 \%$ seed moisture by using nuclear magnetic resonance (Oxford Analytical Instruments Limited, Oxon, England).

Data Analysis. Data were analyzed using multivariate analyses of covariance (SAS Institute 2003). Analyses of covariance were used to account for and eliminate effects of prefoliar treatment ratings on change in flea beetle feeding injury across dates after treatments. Treatment means were compared by multiple $t$-test obtained by least square means statement of GLM at the 0.05 level (SAS Institute 2003). Main and interaction effects of year by location on flea beetle feeding injury ratings and yields were determined using PROC MIXED procedure (SAS Institute 2003). Year, location, and year $\times$ location interaction were all fixed effects.

Economic Analysis. Partial budget analyses were used to determine economic potential of substituting ecorational insecticides for a standard chemical insecticide to control crucifer flea beetle in canola at each location (Kay et al. 2004). Revenues and costs for each experimental treatment were computed and compared with determine net change in profit when ecorational insecticides are used as alternatives for a standard chemical insecticide. Revenues for each experimental treatment were calculated by multiplying total seed weight per hectare by the 14-yr average price of canola for low, medium, and high market prices of $\$ 0.15 / \mathrm{kg}, \$ 0.22 / \mathrm{kg}$, and $\$ 0.29 / \mathrm{kg}$. When using a range of canola prices, results show how sensitive changes in net profits are to changes in crop market prices. Cost for each treatment was calculated using the current market price for individual insecticide products. The current market price for field application rates were Helix XTra, \$18.53/ha; SpinTor, 
Table 1. Crucifer flea beetle feeding injury to seedling canola treated with ecorational and chemical insecticides in North Dakota

\begin{tabular}{|c|c|c|c|c|c|c|c|c|c|c|c|c|}
\hline \multirow{3}{*}{ Insecticide treatment } & \multicolumn{12}{|c|}{ Minot } \\
\hline & \multicolumn{3}{|c|}{2001} & \multicolumn{3}{|c|}{2002} & \multicolumn{3}{|c|}{2003} & \multicolumn{3}{|c|}{2004} \\
\hline & $\mathrm{PFA}^{a}$ & $\begin{array}{c}7 \\
\text { DAFA }^{b}\end{array}$ & $\begin{array}{c}14 \\
\text { DAFA }^{c}\end{array}$ & PFA & $\begin{array}{c}7 \\
\text { DAFA }\end{array}$ & $\begin{array}{c}14 \\
\text { DAFA }\end{array}$ & PFA & $\begin{array}{c}7 \\
\text { DAFA }\end{array}$ & $\begin{array}{c}14 \\
\text { DAFA }\end{array}$ & PFA & $\begin{array}{c}7 \\
\text { DAFA }\end{array}$ & $\begin{array}{c}14 \\
\text { DAFA }\end{array}$ \\
\hline & \multicolumn{12}{|c|}{ Flea beetle injury rating ${ }^{d}$} \\
\hline Untreated control & 1.7 & $3.7 \mathrm{~cd}$ & $4.6 \mathrm{c}$ & $3.5 \mathrm{bcd}$ & $5.3 \mathrm{~b}$ & $5.0 \mathrm{cde}$ & 2.5 & $3.6 \mathrm{~b}$ & $3.9 \mathrm{~b}$ & 1.1 & $3.7 \mathrm{~cd}$ & $3.3 \mathrm{~cd}$ \\
\hline Helix XTra (15.03 ml/kg seed) & $\mathrm{ND}^{e}$ & ND & ND & $2.2 \mathrm{a}$ & $2.7 \mathrm{a}$ & $3.7 \mathrm{a}$ & 1.1 & $1.9 \mathrm{a}$ & $2.3 \mathrm{a}$ & 1.0 & $3.3 \mathrm{a}$ & $2.6 \mathrm{a}$ \\
\hline Capture $(0.51 \mathrm{ml} /$ liter $)$ & 1.5 & $1.7 \mathrm{a}$ & $3.1 \mathrm{a}$ & 3.8bcdef & $4.7 \mathrm{~b}$ & $3.8 \mathrm{ab}$ & 2.1 & $3.6 \mathrm{~b}$ & $2.5 \mathrm{a}$ & 1.1 & $3.4 \mathrm{ab}$ & $2.8 \mathrm{ab}$ \\
\hline SpinTor (1.56 ml/liter) & 1.9 & $3.0 \mathrm{bc}$ & $3.9 \mathrm{~b}$ & $4.3 \mathrm{f}$ & $5.0 \mathrm{~b}$ & $4.9 \mathrm{cde}$ & 1.3 & $3.8 \mathrm{bc}$ & $3.8 b$ & 1.2 & $3.7 \mathrm{~cd}$ & $3.1 \mathrm{bc}$ \\
\hline SpinTor (2.34 ml/liter) & 1.8 & $2.4 \mathrm{ab}$ & $3.9 \mathrm{~b}$ & 4.1def & $5.0 \mathrm{~b}$ & $4.5 \mathrm{~cd}$ & 2.2 & $4.0 \mathrm{bc}$ & $3.9 \mathrm{~b}$ & 1.1 & $3.4 \mathrm{abc}$ & $3.1 \mathrm{bc}$ \\
\hline SpinTor (3.13 ml/liter) & 1.6 & $2.8 \mathrm{~b}$ & $3.8 \mathrm{~b}$ & 3.6bcde & $4.8 \mathrm{~b}$ & $4.4 \mathrm{bc}$ & 2.1 & $4.0 \mathrm{bc}$ & $3.8 \mathrm{~b}$ & 1.2 & $3.4 \mathrm{abc}$ & $3.3 \mathrm{~cd}$ \\
\hline BotaniGard (6.25 ml/liter) & 1.5 & $3.9 \mathrm{~cd}$ & $4.7 \mathrm{c}$ & 3.8bcdef & $5.4 \mathrm{~b}$ & $5.3 \mathrm{e}$ & 2.4 & $4.1 \mathrm{bc}$ & $4.2 \mathrm{~b}$ & 1.1 & $3.6 \mathrm{bcd}$ & $3.3 \mathrm{~cd}$ \\
\hline BotaniGard (9.37 ml/liter) & 2.0 & $4.3 \mathrm{~d}$ & $4.9 \mathrm{c}$ & $3.7 \mathrm{bcde}$ & $5.5 \mathrm{~b}$ & $5.4 \mathrm{e}$ & 1.8 & $4.3 \mathrm{bc}$ & $4.1 \mathrm{~b}$ & 1.2 & $3.7 \mathrm{~cd}$ & $3.4 \mathrm{~cd}$ \\
\hline BotaniGard (12.5 ml/liter) & 1.4 & $3.8 \mathrm{~cd}$ & $4.8 \mathrm{c}$ & $3.9 \mathrm{cdef}$ & $5.2 \mathrm{~b}$ & $5.2 \mathrm{de}$ & 2.8 & $4.6 \mathrm{c}$ & $4.2 \mathrm{~b}$ & 1.2 & 3.6abcd & $3.4 \mathrm{~cd}$ \\
\hline Neemix (1.56 ml/liter) & 1.8 & $3.9 \mathrm{~cd}$ & $4.6 \mathrm{c}$ & $4.3 \mathrm{ef}$ & $5.3 \mathrm{~b}$ & $4.9 \mathrm{cde}$ & 1.7 & $4.4 \mathrm{bc}$ & $4.1 \mathrm{~b}$ & 1.2 & 3.6abcd & $3.4 \mathrm{~cd}$ \\
\hline Neemix (3.13 ml/liter) & 1.6 & $3.9 \mathrm{~cd}$ & $4.5 \mathrm{c}$ & $3.7 \mathrm{bcdef}$ & $5.2 \mathrm{~b}$ & $5.0 \mathrm{cde}$ & 1.7 & $4.3 \mathrm{bc}$ & $4.2 \mathrm{~b}$ & 1.1 & $3.6 \mathrm{bcd}$ & $3.6 \mathrm{~d}$ \\
\hline Neemix (6.25 ml/liter) & 1.7 & $4.2 \mathrm{~d}$ & $5.0 \mathrm{c}$ & $3.4 \mathrm{bc}$ & $5.1 b$ & 5.1cde & 1.5 & $4.4 \mathrm{bc}$ & $4.1 \mathrm{~b}$ & 1.2 & 3.6abcd & $3.4 \mathrm{~cd}$ \\
\hline Surround (37.45 g/liter) & 1.6 & $4.6 \mathrm{~d}$ & $5.0 \mathrm{c}$ & $3.2 \mathrm{~b}$ & $5.4 \mathrm{~b}$ & 5.1de & 2.4 & $4.6 \mathrm{c}$ & $4.3 \mathrm{~b}$ & 1.1 & $3.7 \mathrm{~d}$ & $3.3 \mathrm{~cd}$ \\
\hline Surround (93.46 g/liter) & 1.7 & $4.2 \mathrm{~d}$ & $4.9 \mathrm{c}$ & $3.4 \mathrm{bc}$ & $5.4 \mathrm{~b}$ & $5.0 \mathrm{cde}$ & 1.7 & $4.4 \mathrm{bc}$ & $4.4 \mathrm{~b}$ & 1.1 & $3.6 \mathrm{bcd}$ & $3.3 \mathrm{~cd}$ \\
\hline Surround (149.8 g/liter) & 1.5 & $4.4 \mathrm{~d}$ & $4.9 \mathrm{c}$ & $3.6 \mathrm{bcd}$ & $5.3 \mathrm{~b}$ & $5.5 \mathrm{e}$ & 2.1 & $4.5 \mathrm{c}$ & $4.3 \mathrm{~b}$ & 1.1 & 3.6abcd & $3.4 \mathrm{~cd}$ \\
\hline
\end{tabular}

Means within a column followed by the same letter are not significantly different at $P<0.05$.

${ }^{a}$ PFA, prefoliar application, $\approx 7 \mathrm{~d}$ after planting.

${ }^{b} 7$ DAFA, days after foliar application, $\approx 14 \mathrm{~d}$ after planting.

${ }^{c} 14$ DAFA, days after foliar application, $\approx 21 \mathrm{~d}$ after planting.

${ }^{d}$ Flea beetle feeding injury rating based on a scale of $1-6 ; 1,0-3$ pits per seedling $(\approx 0 \%$ feeding injury); $2,4-9$ pits $(\approx 10 \%$ feeding injury); $3,10-15$ pits ( $\approx 25 \%$ feeding injury); $4,16-25$ pits ( $\approx 50 \%$ feeding injury); $5,>25$ pits ( $\approx 75 \%$ feeding injury); and 6 , dead seedling.

${ }^{e} \mathrm{ND}$, no data as Helix XTra was not included in the 2001 study.

$\$ 40.71 /$ ha; BotaniGard, $\$ 73.58 /$ ha; Neemix, $\$ 53.13 /$ ha; and Surround, $\$ 9.88 /$ ha. Revenues and costs for experimental treatments were entered into partial budgets to determine the net change in profit.

\section{Results}

Analysis of variance (ANOVA) of Main and Interaction Effects. Injury ratings at prefoliar application (PFA) and $7 \mathrm{~d}$ after foliar application (DAFA) varied with year, location, and year $\times$ location interaction $($ PFA: $F=513 ; \mathrm{df}=3,540 ; P<0.0001 ; F=23.10 ; \mathrm{df}=$ 2,$540 ; P<0.0001 ; F=31.32 ; \mathrm{df}=1,540 ; P<0.0001$; and 7 DAFA: $F=46.30 ; \mathrm{df}=3,541 ; P<0.0001 ; F=$ 118.85 ; df $=2,541 ; P<0.0001 ; F=14.48 ; \mathrm{df}=1,541$; $P=0.0002)$. At $14 \mathrm{~d}$ after foliar application, only year and location varied $(F=100.11 ; \mathrm{df}=3,541 ; P<0.0001$; $F=30.10 ; \mathrm{df}=2,541 ; P<0.0001)$. Seed yield and percentage of oil varied by year and location (seed: $F=198.07 ; \mathrm{df}=3,453 ; P<0.0001 ; F=49.17 ; \mathrm{df}=2$, 453; $P<0.0001$; and oil: $F=24.49 ; \mathrm{df}=2,335 ; P<$ $0.0001 ; F=49.35 ; \mathrm{df}=1,335 ; P<0.0001)$. Year $\times$ location interactions for seed yield and oil could not be determined by SAS due to uneven replication among years and locations for the 4-yr study period.

Injury Ratings. As expected, injury levels and yield varied from year to year, and site to site, but trends in the treatment effects were consistent among years and sites. Across the years and locations, the seed treatment Helix XTra and Capture resulted in the lowest flea beetle feeding injury after 14 DAFA (NCREC 2001: $F=4.80 ; \mathrm{df}=26,136 ; P<0.0001 ; 2002$, PFA: $F=$ $5.10 ; \mathrm{df}=14,42 ; P<0.0001 ; 7$ and 14 DAFA: $F=2.60$; $\mathrm{df}=28,84 ; P=0.0083 ; 2003,7$ DAFA: $F=5.22 ; \mathrm{df}=$ 28,$116 ; P<0.0001 ; 2004,7$ and 14DAFA: $F=1.90 ; \mathrm{df}=$
28,$146 ; P=0.0077 ;$ LREC 2002: $F=3.32 ; \mathrm{df}=14,42$; $P=0.0013$; and CREC 2003, 7 and 14 DAFA: $F=2.34$; $\mathrm{df}=28,144 ; P=0.0006 ; 2004: F=3.17 ; \mathrm{df}=28,146$; $P=0.0480$ ) (Tables 1 and 2). Among the foliar treatments injury ratings due to Capture was lower but not always significant compared with SpinTor. BotaniGard, Neemix, and Surround treatments resulted in high injury ratings. This indicates that these ecorationals were not effective for controlling flea beetles.

Yield Traits. Helix XTra and Capture treatments resulted in greater yields than the control (NCREC 2001: $F=4.27 ; \mathrm{df}=13,65 ; P<0.0001 ; 2002: F=1.73$; $\mathrm{df}=14,42 ; P=0.0086 ; 2003,7$ DAFA: $F=5.22 ; \mathrm{df}=$ 28,$116 ; P<0.0001$; LREC 2002: $F=12.50 ; \mathrm{df}=14,42$; $P<0.0001$; and CREC 2003: $F=2.44 ; \mathrm{df}=14,70 ; P=$ $0.0074 ; 2004: F=3.17 ; \mathrm{df}=28,146 ; P=0.0480$ ) (Table $3)$. Among the foliar treatments seed yields generally did not differ much when Capture and SpinTor are compared. Capture produced yields that were greater than all ecorational treatments (CREC 2003: $F=2.44$; $\mathrm{df}=14,70 ; P=0.0074)$ or when compared with the control (CREC 2004: $F=3.17 ; \mathrm{df}=28,146 ; P=0.0480$ ) (Table 3). Capture and SpinTor, and Helix XTratreated canola produced significantly higher percentage of oil than the untreated check in percent oil yield comparisons (NCREC 2001: $F=3.43 ; \mathrm{df}=13,65 ; P=$ 0.0005; LREC 2002: $F=3.34$; $\mathrm{df}=14,42 ; P=0.0012$; and CREC 2004: $F=9.06$; $\mathrm{df}=14,70 ; P<0.0001$ ) (Table 4).

Economic Comparisons. At Minot, when ecorational insecticides were substituted for Helix XTra, net decreases in profits were $\$ 65.68$ to $\$ 265.84 /$ ha compared with decreases in net profits of $\$ 1.79$ to $\$ 5.03 /$ ha and an increase in net profit of $\$ 1.46 /$ ha for Capture (Table 5). The untreated control resulted in net losses 
Table 2. Crucifer flea beetle feeding injury to seedling canola treated with ecorational and chemical insecticides in North Dakota

\begin{tabular}{|c|c|c|c|c|c|c|c|c|c|}
\hline \multirow{3}{*}{ Insecticide treatment } & \multicolumn{3}{|c|}{ Langdon } & \multicolumn{6}{|c|}{ Carrington } \\
\hline & \multicolumn{3}{|c|}{2002} & \multicolumn{3}{|c|}{2003} & \multicolumn{3}{|c|}{2004} \\
\hline & $\mathrm{PFA}^{a}$ & $7 \mathrm{DAFA}^{b}$ & $14 \mathrm{DAFA}^{c}$ & PFA & 7 DAFA & 14 DAFA & PFA & 7 DAFA & 14 DAFA \\
\hline & \multicolumn{9}{|c|}{ Flea beetle injury rating ${ }^{d}$} \\
\hline Untreated control & $4.8 \mathrm{~b}$ & $5.8 \mathrm{~d}$ & $5.9 \mathrm{c}$ & 1.1 & $3.0 \mathrm{de}$ & $3.8 \mathrm{~cd}$ & 1.1 & $3.2 \mathrm{bcd}$ & $2.9 \mathrm{bcd}$ \\
\hline Helix XTra (15.03 ml/kg seed) & $2.5 \mathrm{a}$ & $5.1 \mathrm{ab}$ & $4.2 \mathrm{a}$ & 1.1 & $1.8 \mathrm{ab}$ & $2.9 \mathrm{ab}$ & 1.0 & 2.4abcd & $2.6 \mathrm{bcd}$ \\
\hline Capture $(0.51 \mathrm{ml} /$ liter $)$ & $5.2 \mathrm{~b}$ & $4.7 \mathrm{a}$ & $4.4 \mathrm{a}$ & 1.2 & $1.6 \mathrm{a}$ & $2.8 \mathrm{a}$ & 1.0 & $1.7 \mathrm{a}$ & $1.5 \mathrm{a}$ \\
\hline SpinTor (1.56 ml/liter) & $5.8 \mathrm{bc}$ & $5.5 \mathrm{bcd}$ & $4.8 \mathrm{ab}$ & 1.1 & $2.5 \mathrm{~cd}$ & $3.3 \mathrm{bc}$ & 1.1 & 2.8abcd & $2.9 \mathrm{bcd}$ \\
\hline SpinTor (2.34 ml/liter) & $4.9 \mathrm{~b}$ & $5.3 \mathrm{bc}$ & $4.8 \mathrm{ab}$ & 1.1 & $2.4 \mathrm{bc}$ & $3.4 \mathrm{~cd}$ & 1.1 & $2.5 \mathrm{abcd}$ & $2.5 \mathrm{abc}$ \\
\hline SpinTor (3.13 ml/liter) & $5.0 \mathrm{~b}$ & $5.1 \mathrm{ab}$ & $4.5 \mathrm{a}$ & 1.1 & $2.3 \mathrm{bc}$ & $3.3 \mathrm{bc}$ & 1.0 & 2.7abcd & $2.8 \mathrm{bcd}$ \\
\hline BotaniGard (6.25 ml/liter) & $4.6 \mathrm{~b}$ & $5.7 \mathrm{~cd}$ & $5.6 \mathrm{bc}$ & 1.1 & $2.4 \mathrm{bcd}$ & $3.5 \mathrm{~cd}$ & 1.0 & $2.1 \mathrm{ab}$ & $2.1 \mathrm{ab}$ \\
\hline BotaniGard (9.37 ml/liter) & $4.9 \mathrm{~b}$ & $6.0 \mathrm{~d}$ & $5.8 \mathrm{c}$ & 1.1 & $2.3 \mathrm{bc}$ & $3.5 \mathrm{~cd}$ & 1.0 & $2.3 \mathrm{abc}$ & $2.7 \mathrm{bcd}$ \\
\hline BotaniGard (12.5 ml/liter) & $5.1 \mathrm{~b}$ & $5.9 \mathrm{~d}$ & $5.5 \mathrm{bc}$ & 1.2 & $2.4 \mathrm{bcd}$ & $3.5 \mathrm{~cd}$ & 1.0 & $2.7 \mathrm{abcd}$ & $2.9 \mathrm{bcd}$ \\
\hline Neemix (1.56 ml/liter) & $4.8 \mathrm{~b}$ & $5.9 \mathrm{~d}$ & $5.8 \mathrm{c}$ & 1.0 & $2.5 \mathrm{~cd}$ & $3.6 \mathrm{~cd}$ & 1.1 & $2.3 \mathrm{abc}$ & $2.4 \mathrm{abc}$ \\
\hline Neemix (3.13 ml/liter) & $6.0 \mathrm{c}$ & $6.0 \mathrm{~d}$ & $6.0 \mathrm{c}$ & 1.0 & $2.6 \mathrm{bcd}$ & $3.6 \mathrm{~cd}$ & 1.1 & 2.4abcd & $2.4 \mathrm{abc}$ \\
\hline Neemix (6.25 ml/liter) & $5.1 \mathrm{~b}$ & $5.9 \mathrm{~d}$ & $5.7 \mathrm{c}$ & 1.2 & $2.5 \mathrm{~cd}$ & $3.6 \mathrm{~cd}$ & 1.0 & 2.6abcd & $2.6 \mathrm{bcd}$ \\
\hline Surround (37.45 g/liter) & $5.1 \mathrm{~b}$ & $5.9 \mathrm{~d}$ & $5.8 \mathrm{c}$ & 1.1 & $2.4 \mathrm{bc}$ & $3.8 \mathrm{~d}$ & 1.1 & $3.3 \mathrm{~cd}$ & $3.2 \mathrm{~cd}$ \\
\hline Surround (93.46 g/liter) & $5.2 \mathrm{~b}$ & $5.9 \mathrm{~d}$ & $5.7 \mathrm{c}$ & 1.2 & $2.6 \mathrm{bcd}$ & $3.6 \mathrm{~cd}$ & 1.1 & $2.8 \mathrm{abcd}$ & $2.9 \mathrm{bcd}$ \\
\hline Surround (149.8 g/liter) & $5.3 \mathrm{~b}$ & $6.0 \mathrm{~d}$ & $5.7 \mathrm{c}$ & 1.1 & $3.2 \mathrm{e}$ & $3.8 \mathrm{~d}$ & 1.2 & $3.5 \mathrm{~d}$ & $3.5 \mathrm{~d}$ \\
\hline
\end{tabular}

Means within a column followed by the same letter are not significantly different at $P<0.05$.

${ }^{a}$ PFA, prefoliar application, at $\approx 7 \mathrm{~d}$ after planting.

$b 7$ DAFA, days after foliar application, at $\approx 14 \mathrm{~d}$ after planting.

${ }^{c} 14$ DAFA, days after foliar application, at $\approx 21 \mathrm{~d}$ after planting.

${ }^{d}$ Flea beetle feeding injury rating based on a scale of $1-6 ; 1,0-3$ pits per seedling $(\approx 0 \%$ feeding injury $) ; 2,4-9$ pits $(\approx 10 \%$ feeding injury); $3,10-15$ pits ( $\approx 25 \%$ feeding injury); $4,16-25$ pits ( $\approx 50 \%$ feeding injury); $5,>25$ pits ( $\approx 75 \%$ feeding injury); and 6 , dead seedling.

of $\$ 53.29$ to $\$ 120.33 /$ ha, and these losses were less compared with the ecorational insecticides but greater than for Capture. Capture resulted in the lowest net change in profits among the treatments at low $(t=2.1604, \mathrm{df}=13, P<0.0007)$, at medium $(t=$ $2.1604, \mathrm{df}=13, P=0.0007)$, at high $(t=2.1604, \mathrm{df}=$ $13, P=0.0036)$ canola seed prices, respectively. At Carrington, replacing the seed treatment with the foliar treatment Capture against crucifer flea beetle resulted in net economic gains. Net change in profits ranged from $\$ 31.67$ to $\$ 53.69 /$ ha (Table 5). When ecorational insecticides or no treatment were substituted for a seed treatment, net economic losses ranged from $\$ 22.18$ to $\$ 170.15 /$ ha (Table 5). Among the foliar treatments, Capture resulted in the highest net change in profits at low, medium and high canola seed prices $(t=2.1604, \mathrm{df}=13, P<0.0001 ; t=2.1604, \mathrm{df}=13$, $P<0.0001$; and $t=2.1604$, $\mathrm{df}=13, P=0.0004)$. Economic comparisons were not done at Langdon, because the study at the Langdon location was conducted for only 1 yr.

\section{Discussion}

Results from this study indicate that flea beetle injury was reduced when Helix XTra, Capture, and the ecorational insecticide SpinTor were used. However, SpinTor was less effective than the conventional in-

Table 3. Canola seed yield after treatment of seedlings with ecorational and chemical insecticides for control of $P$. cruciferae in North Dakota

\begin{tabular}{|c|c|c|c|c|c|c|}
\hline \multirow{2}{*}{ Insecticide treatment } & \multicolumn{3}{|c|}{ Minot } & \multirow{2}{*}{$\begin{array}{l}\text { Langdon } \\
2002\end{array}$} & \multicolumn{2}{|c|}{ Carrington } \\
\hline & 2001 & 2002 & 2003 & & 2003 & 2004 \\
\hline & \multicolumn{6}{|c|}{$\mathrm{kg} / \mathrm{ha}$} \\
\hline Untreated control & $1,666.0 \mathrm{cde}$ & $55.9 \mathrm{bc}$ & $412.5 b$ & $70.4 \mathrm{~g}$ & $1079.0 \mathrm{~cd}$ & $1343.4 \mathrm{c}$ \\
\hline Helix XTra (15.03 ml/kg seed) & $\mathrm{ND}^{a}$ & $264.1 \mathrm{a}$ & $1,161.9 \mathrm{a}$ & $2,364.5 \mathrm{a}$ & $1,359.2 \mathrm{ab}$ & $1,605.9 \mathrm{abc}$ \\
\hline Capture $(0.51 \mathrm{ml} /$ liter $)$ & $2,714.6 \mathrm{a}$ & 183.1ab & $1,150.3 \mathrm{a}$ & $1,775.2 \mathrm{~b}$ & $1,520.6 \mathrm{a}$ & $1,754.7 \mathrm{a}$ \\
\hline SpinTor (1.56 ml/liter) & $2,319.4 \mathrm{abc}$ & $141.7 \mathrm{abc}$ & $678.5 \mathrm{ab}$ & $1,023.0 \mathrm{~cd}$ & $1,142.2 \mathrm{bcd}$ & $1,486.9 \mathrm{abc}$ \\
\hline SpinTor (2.34 ml/liter) & 2,313.0abc & $140.7 \mathrm{abc}$ & $615.3 \mathrm{~b}$ & $748.3 \mathrm{cde}$ & $1,163.6 \mathrm{bcd}$ & $1,650.5 \mathrm{abc}$ \\
\hline SpinTor (3.13 ml/liter) & $2,601.5 \mathrm{ab}$ & $80.0 \mathrm{bc}$ & $734.0 \mathrm{ab}$ & $1,229.8 \mathrm{bc}$ & $1,132.6 \mathrm{bcd}$ & $1,475.4 \mathrm{abc}$ \\
\hline BotaniGard (6.25 ml/liter) & $1,876.8 \mathrm{cde}$ & $146.5 \mathrm{abc}$ & $643.1 b$ & $141.7 \mathrm{fg}$ & $1,197.6 \mathrm{bcd}$ & $1,663.9 \mathrm{ab}$ \\
\hline BotaniGard (9.37 ml/liter) & $1,338.3 \mathrm{e}$ & $132.0 \mathrm{abc}$ & $298.4 \mathrm{~b}$ & $124.6 \mathrm{~g}$ & $1,138.0 \mathrm{bcd}$ & $1,412.2 \mathrm{bc}$ \\
\hline BotaniGard (12.5 ml/liter) & $1,827.9 \mathrm{cde}$ & $88.7 \mathrm{bc}$ & $390.9 b$ & $82.5 \mathrm{~g}$ & $1,294.8 \mathrm{abc}$ & $1,383.8 \mathrm{bc}$ \\
\hline Neemix (1.56 ml/liter $)$ & $1,534.3 \mathrm{de}$ & $19.3 \mathrm{c}$ & $447.2 \mathrm{~b}$ & $141.7 \mathrm{fg}$ & $992.0 \mathrm{~d}$ & $1,578.3 \mathrm{abc}$ \\
\hline Neemix (3.13 ml/liter) & $2,051.5 \mathrm{bcd}$ & $48.2 \mathrm{bc}$ & $437.2 \mathrm{~b}$ & 690.6cdef & $1,138.0 \mathrm{bcd}$ & $1,419.6 \mathrm{bc}$ \\
\hline Neemix (6.25 ml/liter) & $1,500.9 \mathrm{de}$ & $50.1 \mathrm{bc}$ & $542.8 \mathrm{~b}$ & 193.7efg & $1,298.5 \mathrm{abc}$ & $1,435.9 \mathrm{bc}$ \\
\hline Surround (37.45 g/liter) & $1,528.5 \mathrm{de}$ & $81.9 \mathrm{bc}$ & $353.1 b$ & $554.9 \mathrm{defg}$ & $1,067.6 \mathrm{~cd}$ & $1382.5 \mathrm{bc}$ \\
\hline Surround (93.46 g/liter) & $1,740.5 \mathrm{cde}$ & $98.3 \mathrm{bc}$ & $489.6 \mathrm{~b}$ & $229.9 \mathrm{efg}$ & $1,062.0 \mathrm{~cd}$ & $1,342.4 \mathrm{c}$ \\
\hline Surround (149.8 g/liter) & $1,578.0 \mathrm{de}$ & $78.1 \mathrm{bc}$ & $380.1 b$ & $134.6 \mathrm{fg}$ & $1,114.9 \mathrm{bcd}$ & $1,373.6 \mathrm{bc}$ \\
\hline
\end{tabular}

Means within a column followed by the same letter are not significantly different at $P<0.05$.

${ }^{a}$ ND, no data as Helix XTra was not included in the 2001 study. 
Table 4. Percentage of oil yield from canola seed after treatment of seedlings with ecorational and chemical insecticides for crucifer flea beetle management in North Dakota

\begin{tabular}{|c|c|c|c|c|c|}
\hline \multirow{2}{*}{ Insecticide treatment } & \multicolumn{3}{|c|}{ Minot } & \multirow{2}{*}{$\begin{array}{l}\text { Langdon } \\
2002\end{array}$} & \multirow{2}{*}{$\begin{array}{c}\text { Carrington }^{a} \\
2004\end{array}$} \\
\hline & 2001 & 2002 & 2003 & & \\
\hline & \multicolumn{5}{|c|}{$\% \mathrm{oil}^{b}$} \\
\hline Untreated control & $42.0 \mathrm{e}$ & 42.9 & 41.0 & 31.6de & $46.4 \mathrm{a}$ \\
\hline Helix XTra (15.03 ml/kg seed) & $\mathrm{ND}^{c}$ & 43.0 & 42.3 & $39.7 \mathrm{a}$ & $46.3 \mathrm{a}$ \\
\hline Capture (0.51 ml/liter) & $43.5 \mathrm{a}$ & 39.2 & 42.9 & $38.1 \mathrm{ab}$ & $45.4 \mathrm{bc}$ \\
\hline SpinTor (1.56 ml/liter) & $43.1 \mathrm{abc}$ & 42.7 & 42.9 & 35.6abcd & $45.4 \mathrm{bc}$ \\
\hline SpinTor $(2.34 \mathrm{ml} /$ liter $)$ & 43.0abcd & 39.8 & 42.4 & $37.4 \mathrm{abc}$ & $45.1 \mathrm{c}$ \\
\hline SpinTor (3.13 ml/liter) & $43.2 \mathrm{ab}$ & 40.3 & 43.2 & $36.7 \mathrm{abcd}$ & $45.0 \mathrm{c}$ \\
\hline BotaniGard $(6.25 \mathrm{ml} /$ liter $))$ & $42.2 \mathrm{cde}$ & 38.2 & 42.5 & $29.8 \mathrm{e}$ & $46.0 \mathrm{ab}$ \\
\hline BotaniGard $(9.37 \mathrm{ml} /$ liter $))$ & $41.8 \mathrm{e}$ & 32.2 & 42.3 & $29.8 \mathrm{e}$ & $45.5 \mathrm{bc}$ \\
\hline BotaniGard (12.5 ml/liter) & $41.9 \mathrm{e}$ & 35.8 & 42.6 & 33.5 bcde & $45.2 \mathrm{c}$ \\
\hline Neemix (1.56 ml/liter) & $42.0 \mathrm{e}$ & ND & 41.8 & $31.9 \mathrm{cde}$ & $45.7 \mathrm{abc}$ \\
\hline Neemix (3.13 ml/liter) & $42.4 \mathrm{bcde}$ & 38.7 & 42.1 & 32.9 bcde & $43.8 \mathrm{~d}$ \\
\hline Neemix $(6.25 \mathrm{ml} /$ liter $)$ & $42.0 \mathrm{e}$ & 37.9 & 43.2 & 34.4abcde & $43.8 \mathrm{~d}$ \\
\hline Surround (37.45 g/liter) & $41.9 \mathrm{e}$ & 41.9 & 42.2 & $36.5 \mathrm{abcd}$ & $44.1 \mathrm{~d}$ \\
\hline Surround (93.46 g/liter) & $42.0 \mathrm{e}$ & 35.2 & 42.4 & 34.7abcde & $43.6 \mathrm{~d}$ \\
\hline Surround (149.8 g/liter) & $42.1 \mathrm{de}$ & ND & 42.3 & $31.5 \mathrm{de}$ & $45.0 \mathrm{c}$ \\
\hline
\end{tabular}

Means within a column followed by the same letter are not significantly different at $P<0.05$.

${ }^{a}$ Seed was not tested for percentage of oil in 2003

${ }^{b}$ Percentage of oil was determined by NMR.

${ }^{c}$ ND, no data as Helix XTra was not included in the 2001 study; percentage of oil for the low rate of Neemix and high rate of Surround could not be determined because yields were too low in the 2002 study.

secticides, particularly when beetle populations were relatively high. These results agree with McLeod et al. (2002) who observed fewer eggplant flea beetles, Epitrix fuscula Crotch, on eggplant (Solanum spp.) treated with spinosad, chlorfenapyr, and thiamethoxam. Yields were always greater for chemical insecticide treatments compared with SpinTor, with differences being the smallest $(68-374 \mathrm{~kg} / \mathrm{ha})$ at low levels of flea beetle feeding injury, and greatest (775$1,364 \mathrm{~kg} / \mathrm{ha}$ ) when canola seedling injury was high. Although yield differences between SpinTor and chemical insecticides were relatively small when flea beetle injury levels were low, net losses ranged from
$\$ 47$ to $\$ 151 /$ ha when SpinTor was used as an alternative to the standard chemical insecticides. These results suggest that SpinTor would not be an economical alternative to chemical insecticides.

Knowledge of flea beetle dispersion is very important for furthering our understanding of its ecology and control. Flea beetle dispersal pattern in spring determines the most effective control practices (Lamb 1983). However due to the sudden and unpredictable invasion of flea beetle in spring canola, systemic seed treatments are more effective than foliar sprays (Lamb and Turnock 1982). Ecorational insecticides, in particular SpinTor, seemed to be as

Table 5. Projected net change in profits for ecorational insecticides and a foliar chemical insecticide (Capture) as alternatives to a chemical seed treatment (Helix XTra) for $P$. cruciferae control in canola in North Dakota

\begin{tabular}{|c|c|c|c|c|c|c|}
\hline \multirow{2}{*}{ Foliar insecticide } & \multicolumn{3}{|c|}{ Minot } & \multicolumn{3}{|c|}{ Carrington } \\
\hline & $\mathrm{LP}^{a}$ & $\mathrm{MP}^{b}$ & $\mathrm{HP}^{c}$ & LP & MP & $\mathrm{HP}$ \\
\hline & \multicolumn{6}{|c|}{$\$ /$ ha } \\
\hline Untreated control & $(53.29)^{d} \mathrm{~b}$ & $(86.81) \mathrm{b}$ & $(120.33) \mathrm{bc}$ & $(22.18) \mathrm{b}$ & $(41.17) \mathrm{b}$ & $(60.17) \mathrm{b}$ \\
\hline Capture $(0.51 \mathrm{ml} /$ liter $)$ & $1.46 \mathrm{a}$ & $(1.79) \mathrm{a}$ & $(5.03) \mathrm{a}$ & $31.67 \mathrm{a}$ & $42.53 \mathrm{a}$ & $53.39 \mathrm{a}$ \\
\hline SpinTor (1.56 ml/liter) & $(67.62) \mathrm{bc}$ & $(88.81) \mathrm{b}$ & $(110.02) \mathrm{b}$ & (47.38) bcd & $(59.14) \mathrm{bc}$ & $(70.90) \mathrm{bc}$ \\
\hline SpinTor (2.34 ml/liter) & (92.58) bcd & (116.03) bcd & (139.48) bcd & $(53.66) \mathrm{cd}$ & $(58.94) \mathrm{bc}$ & $(64.23) \mathrm{bc}$ \\
\hline SpinTor $(3.13 \mathrm{ml} /$ liter $)$ & $(108.39) \mathrm{cde}$ & (129.81) bcd & (151.23)bcde & (89.27) ef & $(101.77) \mathrm{cdef}$ & (114.27)bcd \\
\hline BotaniGard (6.25 ml/liter) & $(102.78) \mathrm{cd}$ & (125.06) bcd & (147.33)bcd & $(62.82) \mathrm{de}$ & (66.45)bcd & $(70.08) \mathrm{bc}$ \\
\hline BotaniGard (9.37 ml/liter) & $(166.50) \mathrm{fg}$ & (201.35)ef & (236.19) ef & $(122.95) \mathrm{gh}$ & $(137.47) \mathrm{fg}$ & $(151.99) \mathrm{d}$ \\
\hline BotaniGard ( $12.5 \mathrm{ml} /$ liter $)$ & $(199.59) \mathrm{g}$ & $(232.72) \mathrm{f}$ & $(265.84) \mathrm{f}$ & $(150.10) \mathrm{h}$ & (160.13)g & $(170.15) \mathrm{d}$ \\
\hline Neemix (1.56 ml/liter $)$ & (106.56) cde & (140.15)bcde & (173.73)bcde & (64.21) de & (78.03)bcde & $(91.85) \mathrm{bc}$ \\
\hline Neemix (3.13 ml/liter) & (131.70) def & $(164.62) \mathrm{cde}$ & $(197.54)$ cdef & (91.72) ef & (105.98)def & $(120.24) \mathrm{cd}$ \\
\hline Neemix $(6.25 \mathrm{ml} /$ liter $)$ & (150.21) ef & (179.37) def & (208.53) def & $(105.03) \mathrm{fg}$ & (113.11)ef & (121.18) cd \\
\hline Surround (37.45 g/liter) & (65.68) bc & $(100.36) \mathrm{b}$ & (135.05) bcd & $(29.98) \mathrm{bc}$ & $(48.00) \mathrm{b}$ & $(66.03) \mathrm{bc}$ \\
\hline Surround (93.46 g/liter) & $(69.01) \mathrm{bc}$ & $(98.34) \mathrm{b}$ & (127.67) bcd & (48.20) bcd & (67.83) bcd & $(87.45) \mathrm{bc}$ \\
\hline Surround (149.8 g/liter) & (93.58) bcd & (127.45) bcd & (161.32)bcde & $(56.74) \mathrm{cd}$ & (73.42) bcde & $(90.10) \mathrm{bc}$ \\
\hline
\end{tabular}

Treatment cost (\$/ha): Helix XTra, 18.53; Capture, 10.13; SpinTor: low rate, 40.71; medium rate, 60.86; and high rate, 81.02; BotaniGard: low rate, 73.58; medium rate, 110.36; and high rate, 147.14; Neemix: low rate, 53.13; medium rate, 79.68; and high rate, 106.26; and Surround: low rate, 9.88 ; medium rate, 24.68 ; and high rate, 39.52 . Means within a column followed by the same letter are not significantly different at $P<0.05$.

${ }_{a, b, c}$ LP, MP, HP; 14-yr average lowest, medium, and highest prices for canola seed yield equal $\$ 0.15, \$ 0.22$, and $\$ 0.29 / \mathrm{kg}$, respectively.

${ }^{d}$ Numbers enclosed in parentheses are negative values. 
efficacious as the chemical seed treatment Helix XTra, in 2004. This observation could be attributed to depressed flea beetle activity due to cool and wet weather conditions (Milbrath and Weiss 1995). Under low flea beetle feeding pressure $(<12 \%$ cotyledon injury), the study indicated that ecorational insecticides were only slightly less efficacious than the standard foliar chemical insecticide. The treatments Helix XTra, Capture, and SpinTor may be more efficacious than BotaniGard, Neemix, and Surround due to their mode of action as nerve toxins. These nerve toxins exert lethal action by causing irreversible damage to the nervous system of insects and related organisms.

During both years of moderate flea beetle pressure (2001 and 2003), SpinTor was the only ecorational insecticide that maintained flea beetle feeding at slightly less than that occurring in plots treated with the foliar chemical insecticide for up to 7-d postapplication. This could be attributed to the neurotoxic mode of action of SpinTor (spinosad) and Capture (bifenthrin). Spinosad is the active ingredient in SpinTor, and it is derived from metabolites of a soil bacterium Saccharopolyspora spinosa (Thompson et al. 2000, Sparks et al. 2001). Spinosad has a neurotoxic mode of action involving postsynaptic nicotinic acetylcholine and GABA receptors (Salgado 1997, 1998). Bifenthrin, the active ingredient in Capture, is a member of the pyrethoid family of chemicals, and it is effective as a stomach or contact poison. It affects the central and peripheral nervous system of insects and ultimately causes paralysis (Miller and Salgado 1985). Under moderate flea beetle feeding pressure, all ecorational insecticides were less efficacious than the chemical seed treatment. Helix XTra, containing the active ingredient thiamethoxam, also has stomach and contact activity (Mason et al. 2000, Maienfisch et al. 2001). Thiamethoxam interferes with nicotinic acetylcholine receptors in the insect nervous systems (Maienfisch et al. 2001). It is systemic in plants and has long-term residual activity. This mode of action, systemicity, and residual activity make the seed treatment more efficacious than that of the ecorationals. Because of residual activity, the seed treatment provided protection as the beetles moved into the seedtreated plots, and this protection continued up to $21 \mathrm{~d}$ postplanting (Knodel 2005).

During 2002 at the Minot and Langdon research sites, high levels of flea beetle feeding injury (2.5 injury rating) to seedling canola was recorded before foliar treatments could be applied. Under this high feeding pressure, injury ratings increased to $3.7-5.5$ in the foliar-treated plots with the ecorational insecticides being less efficacious. In the insecticide-treated seed plots, the feeding injury rating increased to an average of 3.5. These observations confirm the results of Maienfisch et al. (2001) that thiamethoxam showed consistent control of flea beetles.

Although SpinTor had the greatest efficacy against crucifer flea beetle feeding among the ecorational insecticides tested, yields for SpinTor-treated plots were substantially lower compared with the standard foliar or seed treatment insecticides. Even at low levels of flea beetle feeding injury, such as at Carrington in 2003 and 2004, yields for SpinTor treatments were $68-213 \mathrm{~kg} / \mathrm{ha}$ and $217-374 \mathrm{~kg} / \mathrm{ha}$ lower than Helix XTra and Capture, respectively.

Substituting ecorational insecticides for either standard insecticide, Capture or Helix XTra, to manage crucifer flea beetle would result in net economic losses. However, net losses were lower for SpinTor than the other ecorational insecticides. This study suggests that SpinTor is an efficacious ecorational insecticide, particularly at low flea beetle populations and feeding pressure. It is possible that the efficacy of SpinTor could be enhanced by reformulating the product with increased amounts of the active ingredient, inclusion of a synergist, penetrative surfactants, or higher use rates. Reduced seed yields and lower economic returns observed for SpinTor in this study suggest that this ecorational insecticide would not be an economical alternative to chemical insecticides. Economic feasibility of ecorational insecticides could potentially be enhanced by building environmental costs into the cost of chemical pest management (Higley and Wintersteen 1992). This in turn, could decrease differences in net profit between chemical, and ecorational insecticides for flea beetle management in canola.

\section{References Cited}

Brown, W. J. 1967. Notes on the extralimital distribution of some species of Coleoptera. Can. Entomol. 99: 85-93.

Copping, L. G., and J. J. Menn. 2000. Biopesticides: a review of their action, applications and efficacy. Pest Manag. Sci. 56: $651-676$.

Burgess, L. 1977. Flea beetles (Coleoptera: Chrysomelidae) attacking rape crops in the Canadian prairie provinces. Can. Entomol. 109: 21-32.

Gaugler, R. 2004. Nematodes (Rhabditida: Steinernematidae and Heterorhabditidae). (http://www.nysaes.cornell. edu/ent/biocontrol/pathogens/nematodes.html).

Glogoza, P., M. McMullen, R. Zollinger, A. Thostenson, T. Dejong, W. Meyer, N. Schauer, and J. Olson. 2002. Pesticide use and pest management practices for major crops in North Dakota 2000. North Dakota State Univ. Coop. Ext. Serv. Publ. ER-79. North Dakota Sate University, Fargo, ND.

Hajek, A. E., R. S. Soper, D. W. Roberts, T. E. Anderson, K. D. Biever, D. N. Ferro, R. A. Leburn, and R. H. Storch. 1987. Foliar applications of Beauveria bassiana (Balsamo) for control of the Colorado potato beetle, Leptinotarsa decemllineata (Say) (Coleoptera: Chrysomelidae): an overview of pilot test results from the northern United States. Can. Entomol. 119: 959-974.

Higley, L. G., and W. K. Wintersteen. 1992. A novel approach to environmental risk assessment of pesticide as a basis for incorporating environmental costs into economic injury levels. Am. Entomol. 38: 34-39.

Kay, R. D., W. M. Edwards, and P. A. Duffy. 2004. Farm management, 5th ed. McGraw-Hill, New York.

Knodel, J. J. 2005. A risk management approach to crucifer flea beetle (Coleoptera: Chrysomelidae) control in canola. Ph.D. dissertation, North Dakota State University, Fargo, ND. 
Lamb, R. J. 1983. Phenology of flea beetle (Coleoptera: Chrysomelidae) flight in relation to their invasion of canola fields in Manitoba. Can. Entomol. 115: 1493-1502.

Lamb, R. J. 1984. Effects of flea beetles, Phyllotreta cruciferae (Coleoptera: Chrysomelidae), on the survival, growth, seed yield and quality of canola, rape and yellow mustard. Can. Entomol. 116: 269-280.

Lamb, R. J., and W. J. Turnock. 1982. Economics of insecticidal control of flea beetles (Coleoptera: Chrysomelidae) attacking rape in canola. Can. Entomol. 114: 827840.

Liu, T., and P. A. Stansly. 1995. Toxicity and repellency of some biorational insecticides to Bemisia argentifolii on tomato plants. Entomol. Exp. Appl. 74: 137-143.

Maienfisch, P., M. Angst, F. Brandl, W. Fischer, D. Hofer, H. Kayser, W. kobel, A. Rindlisbacher, R. Senn, A. Steinemann, and H. Widmer. 2001. Chemistry and biology of thiamethoxam: a second generation neonicotinoid. Pest Manag. Sci. 57: 906-913.

Mason, G., M. Rancati, and D. Bosco. 2000. The effect of thiamethoxam, a second generation neonicotinoid insecticide, in preventing transmission of tomato yellow leaf curl geminivirus (TYLCV) by the whitefly Bemisia tabaci (Gennadius). Crop Prot. 19: 473-479.

McLeod, P., F. J. Diaz, and D. T. Johnson. 2002. Toxicity, persistence, and efficacy of spinosad, chlorfenapyr, and thiamethoxam on eggplant when applied against the eggplant flea beetle (Coleoptera: Chrysomelidae). J. Econ. Entomol. 95: 331-335.

Milbrath, L. R., and M. J. Weiss. 1995. Influence of tillage system, planting date, and oilseed crucifers on flea beetle populations (Coleoptera: Chrysomelidae). Can. Entomol. 127: 289-293.

Miller T. A., and V. L. Salgado. 1985. The mode of action of pyrethroids on insects, pp. 43-97. In J. P. Leahy [ed.], The pyrethroid insecticides. Taylor \& Francis, London, United Kingdom.

Miranpuri, G. S., and G. G. Khachatourians. 1995. Entomopathogenicity of Beauveria bassiana toward flea beetles, Phyllotreta cruciferae Goeze (Coleoptera: Chrysomelidae). J. Appl. Entomol. 119: 167-170.

Miranpuri, G. S., M. S. Ubhi, M. King, and G. G. Khachatourians. 1992. Efficacy of different strains of entomopathogenic fungus, Beauveria bassiana against blister beetle, Lytta nuttali Say, pp. 91-92. In Proceedings, National Symposium Research Advance on Integrated Pest Management, October 1992, Punjab Agricultural University, Ludhiana, India. India Society for Advancement of Insect Science, Ludhiana, India.

Nowatzki, T. M., and Weiss, M. J. 1997. Effects of simulated and flea beetle injury to cotyledons on growth of drought- stressed oilseed rape, Brassica napus L. Can. J. Plant Sci. 77: 475-481.

Putnam, L. G. 1977. Response of four brassica seed crop species to attack by crucifer flea beetle, Phyllotreta cruciferae. Can. J. Plant Sci. 57: 987-989.

Salgado, V. L. 1997. The modes of action of spinosad and other insect control products. Down to Earth. 52: 35-43.

Salgado, V. L. 1998. Studies on the mode of action of spinosad: insect symptoms and physiological correlates. Pestic. Biochem. Physiol. 60: 91-92.

SAS Institute. 2003. PROC user's manual, version 9.1 ed SAS Institute, Cary, NC

Sparks, T. C., G. D. Thompson, H. A. Kirst, M. B. Hertlein, J. S. Mynderse, J. R. Turner, and T. V. Worden. 1999. Fermentation-derived insect control agents-the spinosyns, pp. 171188. In F. R. Hall and J. J. Menn [eds.], Biopesticides: use and delivery. Humana Press, Totowa, NJ.

Sparks, T. C., G. D. Crouse, and G. Durst. 2001. Natural products as insecticides: the biology, biochemistry and quantitative structure-activity relationships of spinosyns and spinosoids. Pest Manag. Sci. 57: 896-905.

Thomas, P. 2003. Canola growers manual. Canola Council of Canada, Winnipeg, Manitoba, Canada.

Thompson, G. D., S. H. Hutchins, and T. C. Sparks. 1999. Development of spinosad and attributes of a new class of insect control products. (http://ipmworld.umn.edu/ chapters/hutchins2.htm).

Thompson, G. D., R. Dutton, and T. C. Sparks. 2000. Spinosad-a case study: an example from a natural product discovery programme. Pest Manag. Sci. 56: 696-702.

Turnock, W. J., and S. A. Turnbull. 1994. The development of resistance to insecticides by the crucifer flea beetle, Phyllotreta cruciferae (Goeze). Can. Entomol. 123: 13691375.

Ware, G. W. 1989. The pesticide book, 3rd ed. Thompson Publications, Fresno, CA.

Weiss, M. J., P. McLeod, B. G. Schatz, and B. K. Hanson. 1991 The potential for insecticidal management of flea beetles (Coleoptera: Chrysomelidae) attacking canola. J. Econ. Entomol. 84: 1597-1603.

Westdal, P. H., and W. Romanow. 1972. Observations on the biology of the flea beetle Phyllotreta cruciferae (Coleoptera: Chrysomelidae). Manit. Entomol. 6: 35-45.

Wylie, H. G. 1979. Observations on distribution, seasonal life history, and abundance of flea beetles (Coleoptera: Chrysomelidae) that infest rape crops in Manitoba. Can. Entomol. 111: 1345-1353.

Received 23 September 2006; accepted 28 February 2007 